\title{
Evolución del aprovechamiento de los recursos Web 2.0 en los diarios digitales de México, Perú, Venezuela y España
}

Janet Rodríguez Martínez¹. Universidad CEU Cardenal Herrera

Resumen: Se expone en este trabajo el nivel de aprovechamiento y evolución del uso de las prestaciones Web 2.0 por parte de los diarios digitales de cuatro países. En 2006 se constataba, en términos generales, una presencia poco elevada de estas herramientas en las cabeceras de información analizadas, siendo las evidencias de una baja implementación eran mayores en la prensa digital latinoamericana.

Palabras clave:

Web 2.0, ciberperiodismo, Internet, España, Latinoamérica

Abstract:

It is presented the level of development and evolution of the use of Web 2.0 benefits from online newspapers in four countries. In 2006 it was noted, in general, a slightly elevated presence of these tools on the informative sites. Evidence of a low implementation was higher in Latin America's digital media.

Keywords:

Web 2.0, online journalism, Internet, Spain, Latin America

La autora disfruta de una beca FPDI de la Universidad CEU Cardenal Herrera. Este trabajo se desarrolla en el marco del proyecto "Medios de comunicación en el ciberespacio. El impacto de Internet en los medios de comunicación en España y América Latina. Tendencias", financiado por el MICINN BSO2006 15495, dirigido por Elvira García de Torres. 


\section{Introducción}

En 2005, Pavlik resumía la evolución que había experimentado Internet en tres etapas: en primer lugar, y presente todavía en muchas publicaciones, un período en el que los medios se limitan a reproducir las noticias del medio convencional; en segundo lugar, el desarrollo, con fuerza, en los mejores sitios, de webs en las que se ofrecen contenidos originales con hiperenlaces, elementos interactivos, multimedia y posibilidad de personalización; en una tercera etapa, en periodo incipiente y presente en pocos sitios en el último cambio de siglo, se desarrollan medios con información original creada específicamente para la red, que prestan especial atención a todas las posibilidades que esta ofrece.

\section{Diarios españoles y latinoamericanos en la red}

Diversos estudios ponen de manifiesto que el periodismo contextualizado no es todavía una realidad, tanto en el escenario español como latinoamericano. Razones de orden técnico, económico y cultural se apuntan como causas por las que tanto la convergencia como la narración interactiva multimedia o la gestión del contenido generado por los usuarios siguen siendo asignaturas pendientes para los medios digitales.

Franco y Guzmán (2007), al actualizar un estudio de 2004 en el que se examinaba la situación de las redacciones de los diarios latinoamericanos líderes en sus respectivos mercados, no han encontrado una evolución importante: aunque el 74\% de los medios pretendía integrar sus redacciones online e impresa a corto, mediano o largo plazo, solo el $4 \%$ ya estaba totalmente integrado; el número de empleados en las redacciones del periódico digital volvía a ser reducida, con solo 3 periodistas en el $42 \%$ de los medios, y de 4 a 8 en el 24\% de los casos (solo una redacción empleaba a 35 profesionales), y el contenido propio era aún insignificante (se percibía una abundante incorporación de material de origen impreso). Por otra parte, un $68 \%$ de los entrevistados defendía la importancia de las herramientas que permiten generar contenido al usuario, y un 30\% abogaba por la implantación de medidas que bloqueen las aportaciones carentes de sentido ético.

En Perú, los profesionales de los medios refieren algunas razones de la baja explotación del potencial de Internet: problemas económicos, escasos ingresos y acceso incipiente a las nuevas tecnologías (Yezerska, 2008). Estas razones coinciden con los bajos Índices de Preparación en Red (Network Readiness Index) obtenidos por los países latinoamericanos (v. Tabla 1), índices muy inferiores a los recogidos para las economías de mayor desarrollo. 
Tabla 1: Network Readiness Index 2008-2009

\begin{tabular}{|lc|lc|}
\hline País & Puesto & País & Puesto \\
\hline Dinamarca & 1 & Chile & 39 \\
\hline Suecia & 2 & Colombia & 64 \\
\hline Estados Unidos & 3 & México & 67 \\
\hline Suiza & 5 & Argentina & 87 \\
\hline Portugal & 30 & Perú & 89 \\
\hline España & 34 & Venezuela & 96 \\
\hline
\end{tabular}

Fuente: Dutta y Mia (2009)

García de Torres et al (2009) realizan un análisis comparativo del estado del contenido generado por el usuario y de los niveles de control en 24 diarios digitales líderes de ocho países: Argentina, Colombia, México, Perú, Portugal, España, Estados Unidos y Venezuela.

El estudio de las condiciones en las que se producen las contribuciones del usuario y del grado de reconocimiento por parte de los medios ofrece resultados directamente relacionados con la oferta de herramientas Web 2.0: se comprueba, en los espacios producidos por el medio, la alta disponibilidad de Listados de noticias más populares (83,3\%), Blogs de reporteros (75\%), Comentarios en blogs de reporteros (70,8\%) y en notas del medio (70,8\%) y Redes sociales en las noticias (62,5\%).

Es menos evidente, sin embargo, la presencia de herramientas de producción de contenido: Invitación a colgar videos (25\%), Espacio para la publicación de blogs de usuarios (16,6\%), Clasificación de los comentarios a los artículos (116,6\%), Compartir fotos y videos de usuarios (16,6\% y 8,3\%, respectivamente) e Invitación para colgar archivos de audio $(8,3 \%)$.

Cely (2006), por su partes, en un estudio de 30 diarios digitales venezolanos, confirma que el volcado continúa siendo el sistema de mayor concurrencia (63,3\%); solo el 33,3\% de los medios se ajusta a las fórmulas más avanzadas.

\section{Objetivos y metodología}

Los resultados que se presentan en esta nota pertenecen a una investigación que tiene como fin la obtención del grado de doctor. El estudio aborda el desarrollo de la Web 2.0 en el periodismo digital de 
Latinoamérica y España, y tiene como principales objetivos actualizar los resultados obtenidos en investigaciones anteriores y continuar con la línea de análisis comparado de las muestras de los países de ambos continentes. Previamente se ha observado que la difusión en el escenario tradicional parece guardar relación con los recursos de lo medios en su versión online, una situación que vuelve a comprobarse al observar el factor de pertenencia a grupos de comunicación, y que se espera confirmar en esta investigación. Los resultados de investigaciones previas no indican pautas de evolución significativas en los últimos años.

Se emplea un análisis descriptivo estructural, para el que se han seleccionado nueve variables², sobre una muestra que abarca los diarios digitales de México, Perú, Venezuela y España. Las cabeceras españolas seleccionadas son aquellas generalistas que cuentan con edición en papel y digital y cuya difusión es controlada por la OJD. Las fuentes para la obtención de la muestra de diarios latinoamericanos han sido los portales Infoamérica y Prensa Escrita.

Las hipótesis de partida se apoyan en los resultados obtenidos en estudios anteriores. En términos generales, se espera encontrar una evolución similar a la que se recoge en los estudios de la prensa estadounidense realizados por la consultora The Bivings Group (2007, 2008). Estas investigaciones muestran una tendencia general positiva en la incorporación de herramientas 2.0 respecto al año 2006.

\section{Resultados}

$\mathrm{Al}$ analizar las muestras de los países americanos se percibe una evolución significativa en el aprovechamiento de las herramientas 2.0. Esta mejora no incluye El Podcast, el Chat y el Blog de reportero, herramientas que en 2006 ya contaban con una incorporación limitada. Los medios prestan especial atención al recurso de sindicación (RSS), que, sin embargo, está sujeto a restricciones: la mayoría de los diarios ofrecen las noticias solo de manera parcial, lo que obliga a retornar al medio para obtener la información completa; además, el volumen de titulares que se exporta pertenece, generalmente, a la portada o a alguna sección específica, mas no a todas las secciones que conforman el diario.

Los espacios que permiten comentar junto a la noticia, así como los foros, aumentan las posibilidades para el fomento del contenido generado por el usuario. Esto contrasta con la poca presencia de chats y blogs de reportero.

Las variables son: RSS, Más Popular/Leído/Respondido (MP); Video (VI); Podcast (PO); Charla (CH); Blog de Reportero (BR), Comentario en los artículos (C); Marcadores (M) y Foros (F). 
Figura 3: Porcentaje de aprovechamiento en los diarios latinoamericanos

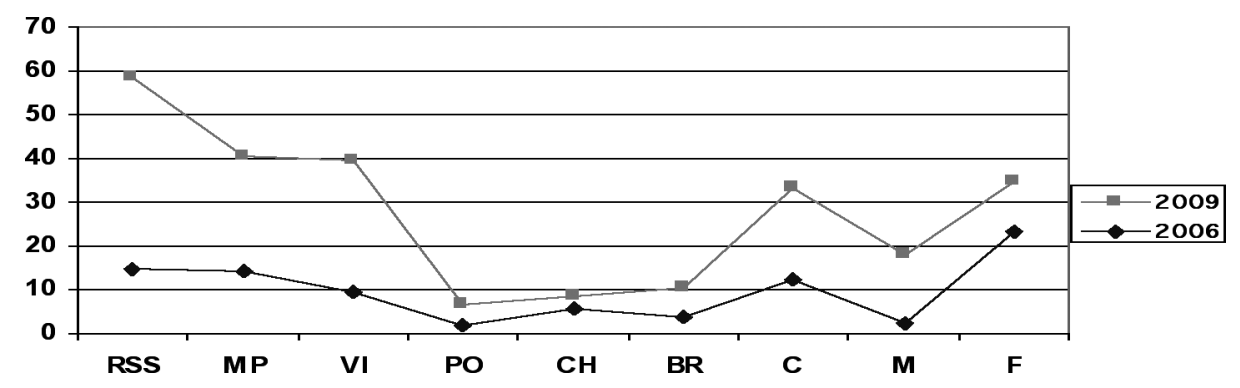

Fuente: Elaboración propia

En términos generales, en 2008 aumentó la incorporación de recursos 2.0 en las cabeceras españolas. Esta situación se evidencia, sobre todo, en determinadas herramientas como el RSS, el Blog de periodista, el Video, la opción Más Popular, y los Comentarios en artículos. El Podcast, por el contrario, mantiene una mínima presencia en las ediciones digitales españolas.

Figura 1: España. Uso de recursos: número de diarios

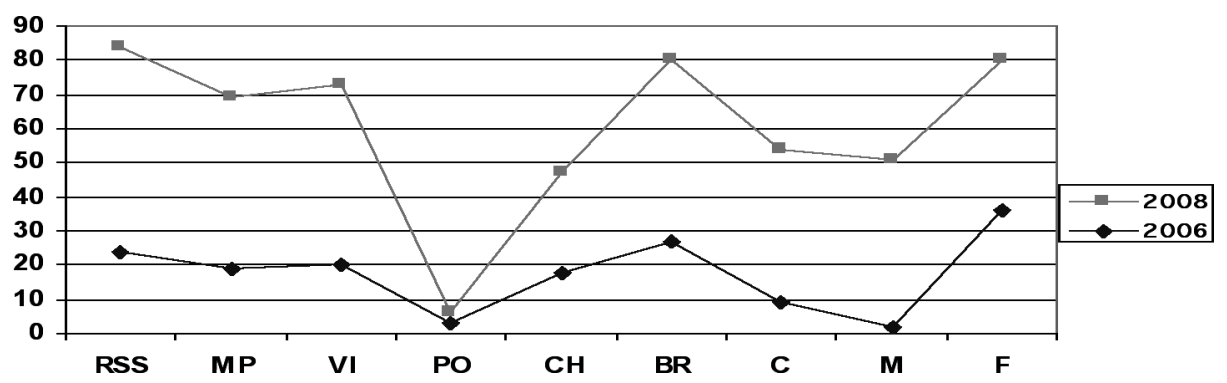

Fuente: Elaboración propia

El 2006 fue un año en el que la mayor concentración de diarios se mantenía en puntuaciones de bajo aprovechamiento (de 0 a 4 recursos). Destacaban El País, El Mundo y La Vanguardia con un uso bastante amplio de los recursos analizados.

La situación de 2006 ha evolucionado, pues la cifra de cabeceras con puntuaciones bajas descendió, en 2008, de un $79 \%$ a un $37 \%$. El 2\% correspondiente a diarios que incorporaban diez o más herramientas pasa ahora al 41\%. En 2008, La Vanguardia comparte una puntuación destacada con más diarios, y otros nueve 
(ocho de ellos pertenecientes a Vocento) la superan por el número de herramientas utilizadas. En febrero de 2008, según datos del estudio Digiworld, El País, ABC, El Correo y La Vanguardia representan unos de los sitios líderes en visitas. Atendiendo a la variable de propiedad, Prisa mantiene su liderazgo en cuanto al aprovechamiento en ambos años. Promecal y Serra, por el contrario, se distancian de modo considerable de los grupos más adaptados a la Web 2.0.

La lista de los diarios “tecnológicos” ha aumentado significativamente. Gran parte de las ediciones han dado un gran salto, al pasar de la nula a la amplia incorporación de las prestaciones de mayor valor tecnológico.

En 2008, el aprovechamiento de las herramientas interactivas tiende al crecimiento, situación que también se refleja en la multimedialidad. En los dos períodos aumenta la disponibilidad de espacios que propician la participación.

Se mantienen las diferencias observadas al comparar las muestras de los cuatro países, y vuelve a obtenerse el mismo orden en los niveles de aprovechamiento. En el segundo período es incluso más evidente el despunte del país europeo con respecto al resto; llega a alcanzar casi un $70 \%$ de aprovechamiento, mientras que las puntuaciones obtenidas en las demás muestras apenas sobrepasan el 30\% (México y Perú) o se mantienen por debajo del $20 \%$ (Venezuela).

Figura 4: Evolución de los niveles de aprovechamiento

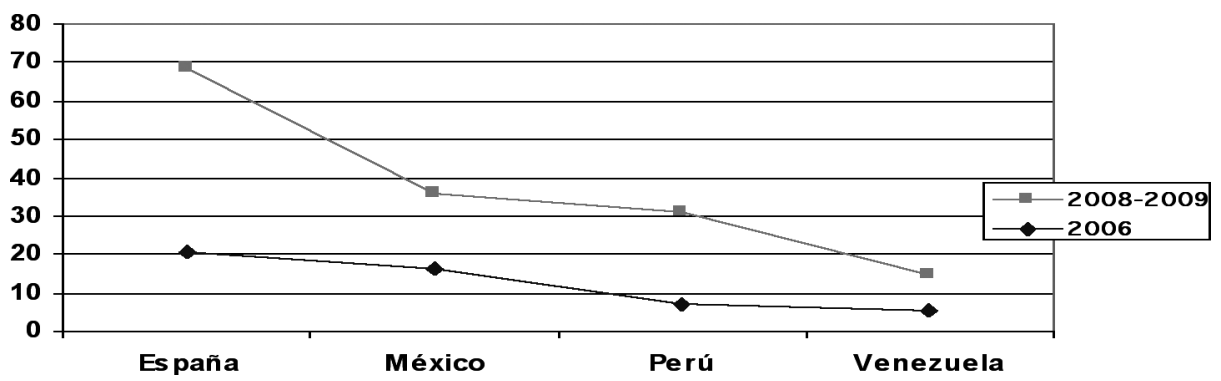

Fuente: Elaboración propia

\section{Conclusiones}

Es significativa la continuidad de las diferencias al contrastar las muestras de España, México, Perú y Venezuela. En términos generales, los diarios americanos responden positivamente a la evolución, pero 
mantienen el retraso evidente con respecto a las cabeceras españolas. El orden que seguían las muestras en el primer año de análisis parece confirmar un patrón que poco ha cambiado en el último período.

Gran parte de las ediciones digitales españolas están adaptándose a la filosofía Web 2.0: se percibe la tendencia de incremento en la utilización de herramientas, lo que coincide con los resultados obtenidos por The Bivings Group en 2007 y 2008 para la muestra de diarios de Estados Unidos.

En un período de dos años, y a pesar del control que ejercen los medios sobre la participación del usuario, este mantiene su entrada en la producción informativa -cada vez más amplia, aunque no siempre adaptada a las preferencias de la audiencia.

Los diarios de España han experimentado un cambio significativo, evolucionando hacia ediciones de un importante valor tecnológico, lejos ya de la tendencia al volcado que se practicaba en los primeros años. En términos cuantitativos, comienzan a acortarse las distancias que separan a los diarios "en primera línea 2.0" del resto de cabeceras con menor incorporación de recursos.

La difusión y la propiedad continúan siendo factores fundamentales. Han demostrado ser determinantes en la implementación de recursos, también en 2008: los diarios con mayor difusión y pertenecientes a ciertos grupos mantienen las puntuaciones más altas.

\section{Referencias bibliográficas}

Cely, A. (2006). "Estructura editorial de los cibermedios de Venezuela”. En Rojano, M. Diez años de periodismo digital en Venezuela 1996-2006. Caracas: CIC-UCAB.

Deuze, M. (2007): “Preparing for an Age of Participatory News”, en Journalism Practice 1 (3): 322-338.

Deuze, M. (2008): “Participation, Remediation, Bricolage: considering principal components of a digital culture”, en The Information Society 2 (22): 63-75.

Domingo, D. et al (2008): "Participatory Journalism Practices in the Media and Beyond: an International Comparative Study of Initiatives in Online Newspapers", en Journalism Practice 2 (3): 326-342.

Dutta, S.; Mia, I. (2009): The Global Information Technology Report 2008-2009. En línea en http://www.insead.edu/v1/gitr/ wef/main/fullreport/index.html, consultado el 12 de julio de 2009.

Franco, G.; Guzmán, J. C. (2004): “Encuesta sobre periodismo de Internet en Latinoamérica”, Sala de Prensa, vol. 3, nº 69. En línea en http://www.saladeprensa.org/art551.htm, consultado el 3 de diciembre de 2006.

Franco, G.; Guzmán, J. C. (2007): Situación del periodismo digital y de los sitios web de periódico de América Latina 2007. En línea en http://www.elpais.com.co/paisonline/especiales/sip/documentos/documento.doc, consultado el 3 de junio de 2009. 
García de Torres, E. (2004): “La especialización en la era de Internet”, en Fernández del Moral, J. (coord.): Periodismo especializado. Madrid: Ariel, pp. 195-218.

García de Torres, E. (2006): "Falling for RSS and Blogs: techs or trend? The Use of Internet by Spain's Newspapers”. Comunicación presentada en la UMN Conference del New Media Research, Universidad de Minnesota, Mineápolis, celebrada el 14 y 15 de septiembre.

García de Torres, E. et al (2006b). “Evolución de las audiencias en Internet 2002-2006”, en Sabés, F; Verón, J. J. (coords): Internet como sinónimo de convergencia tecnológica y mediática. Huesca: Asociación de la Prensa de Aragón, pp. 109-124.

García de Torres, E. et al (2009): UGC Status and Levels of Control in Argentine, Colombian, Mexican, Peruvian, Portuguese, Spanish, US and Venezuelan Online Newspapers. Presentado en el $10^{\text {th }}$ International Symposium on Online Journalism. Universidad de Texas, Austin. En línea en http://online.journalism.utexas.edu/2009/papers/Garciaetal09.pdf, consultado el 5 de julio de 2009.

García de Torres, E. y Rodríguez Martínez, J. (2007): "Herramientas web 2.0 en los diarios digitales de España, México, Venezuela y Perú", en Sabés, Fernando (coord.): Presente y futuro de la comunicación digital. Huesca: Asociación de la Prensa de Aragón, pp. 359-371.

García de Torres, E.; Martínez Martínez, S.; García Cebrián, N. (2006): “Spanish Online Newspaper’s Front Page News Profile: Ownership Strategies and Trends", III COST A20 Conference, Delfos.

García de Torres, E.; Martínez Martínez, S.; Rodríguez Rodríguez, J. (2006): “RSS, marcadores y blogs: estrategias de fidelización en los diarios españoles en Internet”. Comunicación presentada en el I Congreso Internacional de Nuevo Periodismo, Valencia.

García de Torres, E.; Saiz, J.; Cebrián, B.; Berruti, N.; Lavilla, M. J. (2004): "Prensa española digital en primera línea”, 70 años de investigación en comunicación en América Latina, Universidad Nacional de La Plata.

García, E. P. (2007): “Interactivity in Argentinean Online Newsrooms”, en Zer 2007: 7-25.

Hermida, A.; Thurman (2008): "A Clash of Cultures: The Integration of User-Generated Content within Professional Journalistic Frameworks at British Newspaper Websites", en Journalism Practice 2 (3): 343-356.

Keyser, J. D.; Raeymaeckers, K. (2008): "Forcing the Gates of the Fortress: the Growing Impact of Public Participation in Flemish Newsrooms", presentado en Technology, Education and Ethics Conference 2008. En línea en http://theendofjournalism.wdfiles.com/local--files/dekeyseretal/De\%20Keyser\%20\&\%20Raeymaeckers.pdf, consultado el 11 de abril de 2009.

MacGregor, P. (2007): “Tracking the Online Audience”, en Journalism Studies 8 (2): 280-298.

Paulussen, S.; Ugille, P. (2008): "User Generated Content in the Newsroom: Professional and Organisational Constraints in Participatory Journalism”, en Westminster Papers in Communication and Culture 5 (2): 24-41.

Pavlik, J. V. (2005): El periodismo y los nuevos medios. Barcelona: Paidós. 
Sabés, F. y Verón, J. J. (2008): Internet como sinónimo de convergencia tecnológica y mediática. Huesca: Asociación de la Prensa de Aragón.

Singer, J.; Ashman, I. (2008): "Comment Is Free, but Facts Are Sacred. User-Generated Content and Ethical Constructs at The Guardian”, presentado en Media Ethics, Asssociation for Education in Journalism and mass Communication. Chicago, agosto de 2008.

The Bivings Group Report (2007): The Use of the Internet by America's Newspapers. En línea en http://www.bivingsreport.com/wp-content/uploads/2007/08/newspaperstudy_22.pdf, consultado el 11 de junio.

The Bivings Group Report (2008): The Use of the Internet by America's Largest Newspapers. En línea en http://www.bivings.com/thelab/presentations/2008study.pdf, consultado el 13 de mayo de 2009.

Thurman, N. (2008): “Forums for Citizen Journalists? Adoption of User Generated Content Initiatives by Online News Media”, en New Media \& Society 10 (1): 139-157.

Yezerska, L. (2008): Ciberperiodismo en Perú, análisis de los diarios digitales. Lima: Universidad de Piura, Editorial San Marcos.

Zamith, F. (2008a): "A Methodological Proposal to Analyze the News Websites Use of the Potentialities of the Internet”, presentado en el $9^{\text {th }}$ International Symposium on Online Journalism, Universidad de Texas, 2008. En línea en http://online.journalism.utexas.edu/2008/papers/Zamith.pdf, consultado el 2 de febrero de 2009.

Zamith, F. (2008b): Ciberjornalismo. As potencialidades da Internet nos sites noticiosos portugueses. Oporto: Ediçoes Afrontamento. CETAC Media. 